\title{
Thermal annealing of tilted fiber Bragg gratings
}

\author{
Á. González-Vila*a, L. Rodríguez-Cobo ${ }^{\mathrm{b}}$, P. Mégret ${ }^{\mathrm{a}}$, C. Caucheteur ${ }^{\mathrm{a}}$, J. M. López-Higuera ${ }^{\mathrm{b}}$ \\ ${ }^{a}$ Electromagnetism and Telecommunication Department, University of Mons, \\ Boulevard Dolez 31, 7000 - Mons, Belgium \\ ${ }^{b}$ Photonics Engineering Group, University of Cantabria, Edificio I+D+i, \\ Avenida de Los Castros s/n, 39005 - Santander (Cantabria), Spain
}

\begin{abstract}
We report a practical study of the thermal decay of cladding mode resonances in tilted fiber Bragg gratings, establishing an analogy with the "power law" evolution previously observed on uniform gratings. We examine how this process contributes to a great thermal stability, even improving it by means of a second cycle slightly increasing the annealing temperature. In addition, we show an improvement of the grating spectrum after annealing, with respect to the one just after inscription, which suggests the application of this method to be employed to improve saturation issues during the photo-inscription process.
\end{abstract}

Keywords: Optical fiber sensors, fiber Bragg grating, tilted fiber Bragg grating, thermal annealing

\section{INTRODUCTION}

Fiber Bragg gratings (FBGs) are nowadays essential devices when referring to optical fiber sensing. They consist of a local and periodic refractive index modulation of the core of an optical fiber. The index modification along a certain length of fiber gives raise to the reflection of part of the light backwards, and to the transmission of the rest of it towards the remaining part of the link. That is why these structures act as in-fiber optical filters and are used in a wide range of applications ${ }^{1}$.

Among optical gratings, the ones on which we are going to base the discussed work are the tilted fiber Bragg gratings (TFBGs). Unlike uniform gratings, in these structures the modulation pattern of core is angled with respect to the perpendicular to the fiber longitudinal axis. This tilt angle allows the reflected light not only to be confined in the fiber core (Bragg mode), but also to be coupled to the fiber cladding in the form of several modes (cladding mode resonances). Each of the modes propagates inside the fiber cladding with a corresponding effective refractive index value. However, as soon as the refractive index of the medium surrounding the optical fiber reaches a value close to the one of a cladding mode, the mode is no longer totally internally reflected at the cladding outer interface. When this happens, the mode is coupled to the surrounding medium, producing a change on the grating transmitted spectrum. This phenomenon has lead to the exploitation of tilted fiber Bragg gratings as highly sensitive refractometers in the development of several sensing platforms ${ }^{2}$.

For certain applications, fiber gratings are required to withstand working temperatures of hundreds of degrees. However, to achieve a good thermal stability we have to expose them to different treatments, such as regeneration processes or annealing cycles. Regeneration ${ }^{3}$ is perhaps the most discussed technique and the one that typically leads to the highest thermal stability, but it has the counterpart of producing a physical damage to the optical fiber due to the very high temperatures that are used. In our case, we have decided to carry out an annealing process to stabilize the thermal behavior of tilted fiber Bragg gratings and get a deeper knowledge of their evolution, in order to extend the range of temperature in which we are able to use them.

\section{ANNEALING OF THE GRATINGS}

The tilted fiber Bragg gratings that we used in the experiments were photo-inscribed in the core of a hydrogen-loaded single-mode optical fiber from Draka (Bendwright XS) ${ }^{4}$ through the UV radiation produced by a $\mathrm{CW}$ frequency-doubled

*alvaro.gonzalezvila@umons.ac.be; phone +32 (0) 653743 67; fax +32 (0) 653741 99; umons.ac.be/telecom

Sixth European Workshop on Optical Fibre Sensors, edited by Elfed Lewis, Proc. of SPIE Vol. 9916, $991611 \cdot$ • (c) 2016 SPIE · CCC code: 0277-786X/16/\$18 · doi: 10.1117/12.2236928 
Ar-ion laser emitting at $244 \mathrm{~nm}$. We made use of a $1083.31 \mathrm{~nm}$ pitch phase-mask and produced several $6^{\circ}$ tilted Bragg gratings with a length of $24 \mathrm{~mm}$ each, focusing on achieving the most resonance depth possible.

A first characterization was aimed to evaluate the thermal decay of the UV-induced refractive index changes that comprise the gratings. Typically, the way of studying the evolution of uniform gratings with regard to temperature changes has been based on the tracking of the Bragg mode, establishing its wavelength shift and reflectivity change. However, the most remarkable fact when referring to tilted fiber Bragg gratings in the field of optical sensing, is their ability to interact with the medium surrounding the optical fiber by means of their cladding mode resonances ${ }^{5}$. This is therefore important to weigh an analysis that takes into consideration the behavior of these resonances.

We introduced a tilted fiber Bragg grating on the inside of a tubular furnace to study its evolution with respect to time and temperature. The thermal cycle that we set consisted of a stepped ramp with temperature increments of $100{ }^{\circ} \mathrm{C}$ from room temperature to $700{ }^{\circ} \mathrm{C}$ and with remaining periods of 1 hour on each step. In order to interrogate the TFBG, we connected it to a white light source on one side and to an optical spectrum analyzer on the other end in such a way that we measured the device's transmission spectrum. To minimize the errors arisen from the limited resolution of the measurement unit and focus on the cladding mode amplitude evolution as a whole, we computed the area delimited by the TFBG resonances on each instant, obtaining the results depicted on the left side of Figure 1.
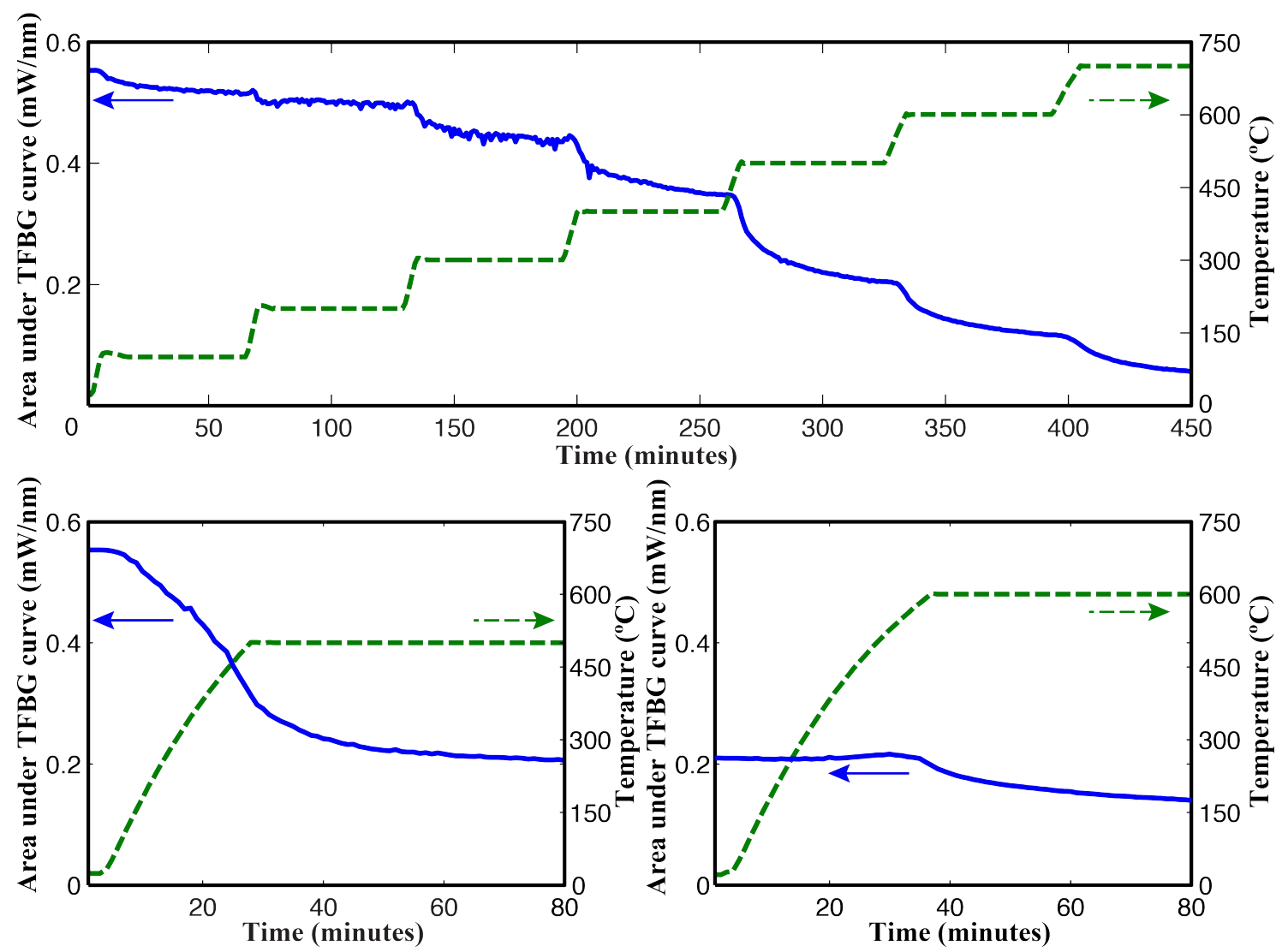

Figure 1. Evolution of the TFBG spectrum while being exposed to the stepped thermal cycle (top), first annealing at $500{ }^{\circ} \mathrm{C}$ (bottom left) and second annealing at $600^{\circ} \mathrm{C}$ (bottom right).

As it can be seen, the area under the TFBG spectrum curve tends to rapidly decrease, due to the reduction of the resonance depth, that is due in turn to the decay of the UV-induced refractive index pattern. In fact, the fall of this area can be approximated by several "power law" functions of time ${ }^{6}$, initiated on the temperature changes produced on every step of the thermal cycle. The thermal behavior of this kind of gratings seems to be similar to the one exhibited by uniform fiber Bragg gratings, so we photo-inscribed another tilted grating and tried to carry out an accelerated annealing, targeting to obtain a thermally stable grating able to keep most of its cladding resonances for sensing proposes. 
On the bottom left part of Figure 1 we illustrate the first annealing we made, consisting of a single-step thermal cycle at $500{ }^{\circ} \mathrm{C}$ during 1 hour. This temperature was chosen based on the previous study, since we could observe a strong decay of the grating area with respect to the starting point, but keeping a reasonable level of resonances still present. What we see is a single decay, since we configured just a single step in temperature, but the same "power law" evolution, as expected. After that, we carried out a second annealing process, identical to the previous one except for an increase of $100{ }^{\circ} \mathrm{C}$ on the set temperature. We can observe how the area under the curve decays just the proportional part corresponding to this increment of $100{ }^{\circ} \mathrm{C}$. This gives us enough evidences to support the validity of this accelerated annealing process, proving the major dependency of temperature over time on the decay of the UV-induced pattern photo-inscribed in the fiber core.

\section{RESULTS}

The annealing procedures described before were followed by their corresponding thermal stability tests, as we can see on the top part of Figure 2. The test on the left was carried out just after annealing the grating at $500{ }^{\circ} \mathrm{C}$. It can be seen that the general stability of the cladding mode resonances, reflected as well on the computed area under the curve, was pretty good, both for temperature changes and for the part of the cycle where the temperature remained constant. The overall decay, calculated by a linear fit, was $1.87 \times 10^{-4} \mathrm{~mW} / \mathrm{nm} \mathrm{h}^{-1}$, which can be considered as an important improvement with respect to the non-annealed tilted grating.
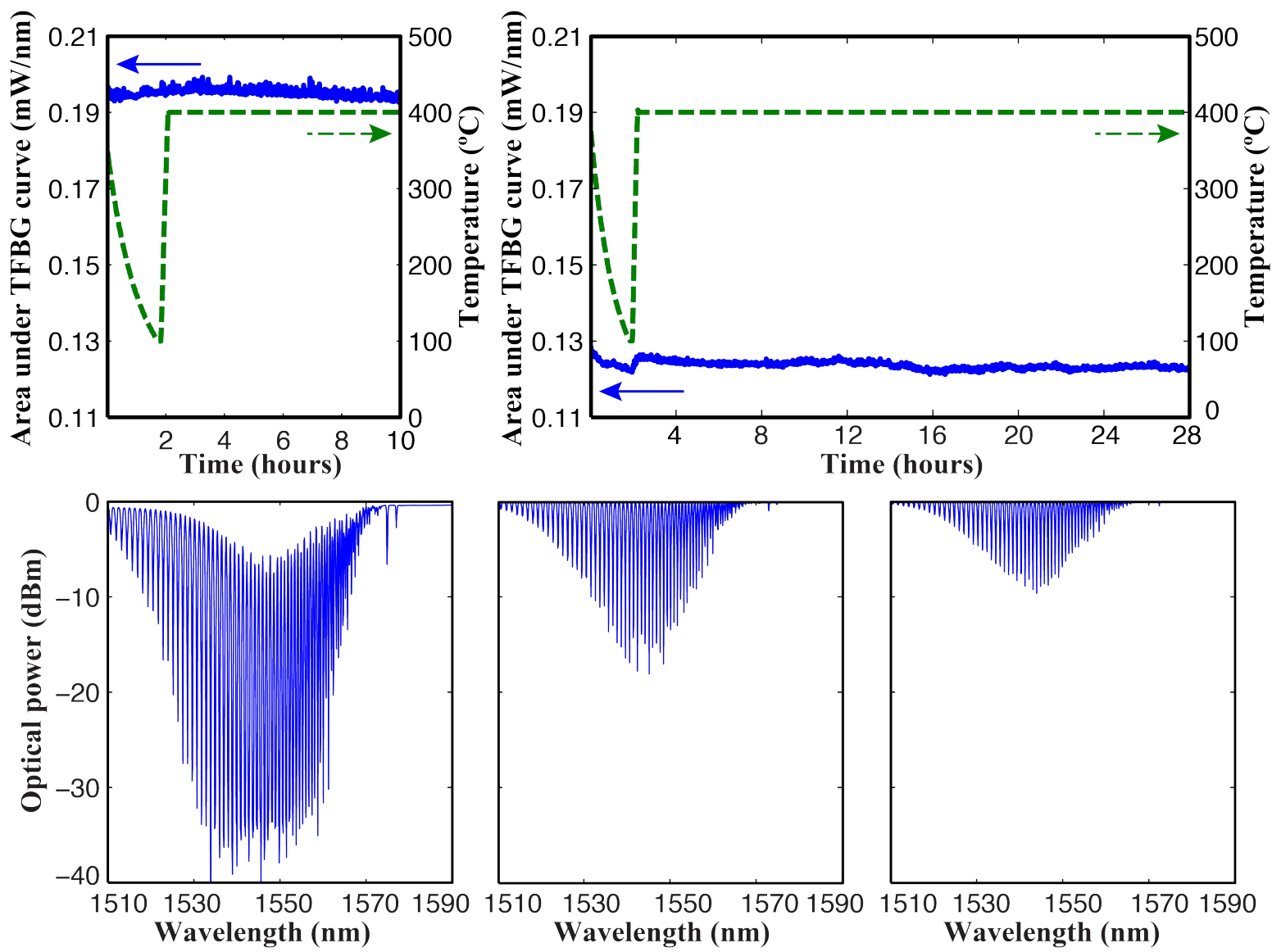

Figure 2. First stability test (top left), second stability test (top right), TFBG spectrum before any annealing (bottom left), after the first annealing (middle) and after the second (bottom right).

In addition to this test, we wanted to compare the differences between the two annealing processes in terms of stability. After annealing the same grating at $600{ }^{\circ} \mathrm{C}$, we performed a longer-term stability test again at $400{ }^{\circ} \mathrm{C}$ obtaining the data on the right. This time, the decay of the computed area resulted $0.96 \times 10^{-4} \mathrm{~mW} / \mathrm{nm} \mathrm{h}^{-1}$. In terms of a comparative 
analysis, it is almost the half of the decay arisen during the previous test. Therefore, it is worth to notice the fact that the higher temperature we use during the annealing process is, the more stable becomes the grating, in terms of cladding mode resonance amplitude

We would also like to highlight the changes produced on the tilted fiber Bragg grating spectrum, as shown on the bottom part of Figure 2. The spectrum on the left corresponds to the grating after the photo-inscription process, before any annealing. As we can see, the grating exhibits very deep cladding mode resonances, as well as a deformation on the upper part of the spectrum, due to the saturation suffered during its manufacture. However, the two following pictures on the right show how the resonances have decreased in depth and at the same time the deformation on the top has completely disappeared. What we have as a result is a more typical TFBG curve, that is, the one corresponding to a grating we could have produced for sensing applications, without forcing the inscription conditions. As expected, the resonances in the last spectrum are shallower than the ones of the spectrum on the middle, since the second annealing produces an additional decay into the computed grating area.

\section{CONCLUSIONS}

We have reported a preliminary study of the decrease of the cladding mode resonance depth produced on a tilted fiber Bragg grating when it is exposed to different temperature conditions. This process is originated on the decay of the UVinduced pattern photo-inscribed during the manufacturing process of the proper grating and sometimes it might lead to the inability to use a device of this kind, specially for a long term period. As we have seen, the decay follows a "power law" evolution, mainly dependent on the working temperature over the exposure time. This is why we have satisfactory carried out an accelerated annealing process based on a single-step ramp at a fixed temperature during only 1 hour.

After the annealing, we have tested the grating performance on demanding temperature conditions, obtaining a high degree of stability. In addition, we have demonstrated in a practical way that, within the limits of resonance preservation, the higher the temperature used for the annealing is, the more stable becomes the grating in terms of cladding mode resonance amplitude. Finally, we have observed that the spectrum of the tilted fiber Bragg grating narrows its resonances after the thermal cycles, with respect to the spectrum it had after its photo-inscription. This modification suggests that the thermal annealing processes might be not only a way of stabilizing a grating for its later use on harsh temperature environments, but also a way of improving the spectrum clearness in order to obtain a higher quality device for a potential demanding application.

\section{ACKNOWLEDGEMENTS}

Á. González-Vila is supported by the F.R.S.-FNRS through a FRIA grant. C. Caucheteur is supported by the F.R.S.FNRS. The authors would also like to thank the financial support from the ERC (European Research Council) Starting Independent Grant PROSPER (grant agreement No. 280161 - http://hosting.umons.ac.be/aspnet/erc-prosper/) and from the project TEC2013-47264-C2-1-R of the Spanish government.

\section{REFERENCES}

1. Kashyap R., "Fiber Bragg Gratings ( $2^{\text {nd }}$ Edition)", Academic press, 2009.

2. Albert J., Shao L. Y., Caucheteur C., "Tilted fiber Bragg grating sensors”, Laser \& Photonics Reviews, 7(1), 83-108 (2013).

3. Wang T., Shao L. Y., Canning J., Cook K., "Temperature and strain characterization of regenerated gratings", Optics letters, 38(3), 247-249 (2013).

4. http://www.prysmiangroup.com/en/business_markets/markets/fibre/downloads/datasheets/SMF---BendBright-XSSingle-Mode-Optical-Fiber.pdf

5. Laffont G., Ferdinand P., "Tilted short-period fibre-Bragg-grating-induced coupling to cladding modes for accurate refractometry", Measurement Science and Technology, 12(7), 765 (2001).

6. Erdogan T., Mizrahi V., Lemaire P. J., Monroe D., "Decay of ultraviolet-induced fiber Bragg gratings", Journal of Applied Physics, 76(1), 73-80 (1994). 